\title{
Ordem dos exercícios físicos aeróbio e com pesos na aptidão física de mulheres acima de 50 anos
}

\author{
Effect of the sequence of aerobic and resistance exercise \\ on physical fitness in women over the age of 50
}

Marcelo Cozzensa da Silva Airton José Rombaldi Anderson Leandro Peres Campos

1 Universidade Federal rior de Educação Física. Pelotas, RS. Brasil.

Recebido em 20/08/08 Revisado em 15/04/09 Aprovado em 14/07/09
Resumo - O treinamento concorrente vem sendo utilizado, frequentemente, embora pouco ainda se saiba sobre o efeito da ordem de execução das atividades na aptidão física de idosas. O objetivo do presente estudo foi o de verificar o efeito da ordem dos exercícios físicos aeróbio e com pesos na aptidão física de mulheres acima de 50 anos. A amostra foi composta por 26 sujeitos do sexo feminino, alocadas aleatoriamente em dois grupos de intervenção: $A_{1}$ (aeróbio e treinamento com pesos) e $M_{1}$ (treinamento com pesos e aeróbio). Foram mensuradas as variáveis peso corporal, estatura, índice de massa corporal (IMC), somatório de dobras cutâneas, flexibilidade, além da força de membros inferiores, lombar e de preensão manual. Como procedimento de análise de dados, utilizou-se a estatística descritiva e ANOVA de uma entrada, utilizando-se nível de significância de 5\%. Os resultados evidenciaram alterações significativas para o grupo $A_{1}$ na força da região lombar $(p=0,01)$ e de membros inferiores $(p=0,0002)$, após as 12 semanas, e para o grupo $M_{1}$ na força de membros inferiores $(p=0,02)$. Com exceção do IMC $(p=0,05)$, não foram encontradas alterações nas medidas antropométricas, de força e flexibilidade entre os grupos no pós teste. Conclui-se que, independentemente da ordem de execução dos exercícios no treinamento concorrente, as mulheres estudadas apresentaram melhora nos índices de força e mantiveram inalterados outros índices, o que, para pessoas em idade avançada, é de fundamental importância, pois isso afeta diretamente a melhoria da qualidade de vida e saúde dessa população.

Palavras-chave: Mulheres; Aptidão física; Envelhecimento; Força muscular; Exercício

Abstract - Concurrent training has been frequently used, although little is known about the effects of the exercise sequence on physical fitness in elderly women. The objective of this study was to determine the effect of the sequence of aerobic and resistance exercise on physical fitness in women over 50 years old. The sample consisted of 26 women randomly divided into two groups: $A_{1}$ (aerobic and resistance training) and $M_{1}$ (resistance and aerobic training). Body weight, height, body mass index (BMI), sum of skinfolds, flexibility, and leg, back and hand grip strength were measured. Descriptive statistics and one-way ANOVA were used for data analysis, adopting a level of significance of 5\%. The results showed significant changes in back strength $(p=0.01)$ and leg strength $(p=0.0002)$ after 12 weeks in group $A_{1}$, and in leg strength $(p=0.02)$ in group $M_{1}$. Except for BMI ( $\left.p=0.05\right)$, no differences in anthropometric measures, strength or flexibility were observed between groups after testing. In conclusion, improvement of strength and the lack of change in the other indices were observed in the women studied, irrespective of the sequence of concurrent training. This finding is important for older adults since it directly affects improvement in the quality of life and health of this population.

Key words: Women; Physical fitness; Aging; Muscle strength; Exercise. 


\section{INTRODUÇÃO}

O envelhecimento humano é caracterizado por uma falência progressiva da eficiência de todos os órgãos e tecidos do individuo em diferentes graus. Consequentemente, o indivíduo experimenta uma perda de suas funções intelectuais e motoras, tornando-se dependente de seus semelhantes para a sua sobrevivência. À medida que vai envelhecendo, o organismo passa por diversas modificações, a velocidade e a quantidade da multiplicação celular vão diminuindo, as células produzem menos enzimas e proteínas ${ }^{1}$.

A diminuição da capacidade locomotora é devida, principalmente, a sarcopenia, que compreende um conjunto de alterações morfofuncionais nos músculos. Nessa perspectiva, enquanto exercícios aeróbios ajudam na prevenção de doenças crônico-degenerativas tais como diabetes tipo II, hipertensão e doenças cardíacas, o treinamento de força, por acrescentar um bom tônus muscular, vem sendo o primeiro método de intervenção para melhorar e manter a independência funcional de pessoas idosas, visto que é nessa fase da vida que se acentuam os acidentes por quedas, causados pela diminuição da força muscular².

A inatividade física e a má alimentação são consideradas importantes causas de debilidade, diminuição da qualidade de vida e morte prematura ${ }^{3}$. Sabe-se que o problema de quedas, por exemplo, está fortemente associado a limitações fisiológicas de força, flexibilidade, equilíbrio e tempo de reação ${ }^{4,5}$. A perda da força muscular é a principal responsável pela deterioração na mobilidade e na capacidade funcional do indivíduo que está envelhecendo ${ }^{6}$. A participação efetiva em um programa de exercícios físicos (aeróbicos e de força) fornece inúmeras respostas favoráveis que contribuem para o envelhecimento saudável. Para Fleck \& Kraemer?, o treinamento de força de alta intensidade $(80 \%$ de $1 \mathrm{RM}$ ) pode ser tolerado e resulta em adaptações positivas nos idosos. A recuperação de uma sessão de treinamento com pesos pode ser mais demorada, mas os resultados são positivos. No entanto, para Lacroix et al. ${ }^{8}$, exercícios aeróbios, como a caminhada, realizada quatro horas por semana, são mais eficazes para diminuir o risco de hospitalização por acidentes cardiovasculares. Por outro lado, Keisor \& Jette ${ }^{9}$, afirmam que a caminhada promove aumento da força e ainda melhora a capacidade aeróbia, implicando um aumento da resistência à fadiga.

Atualmente, não há dúvida dos benefícios trazidos pela prática regular de exercícios físicos à saúde dos praticantes, bem como da importância da utilização de atividades aeróbias e resistidas em sua composição ${ }^{10}$. A grande discussão é em torno da ordem dessas atividades, quando realizadas numa mesma sessão de um programa para a promoção da saúde. Alguns autores sugerem que o treinamento concorrente pode prejudicar o desenvolvimento da força, hipertrofia e potência muscular ${ }^{11-13}$, possivelmente, em função de diferentes adaptações neurais ${ }^{13,14}$. No entanto, a diferença nos resultados parece estar relacionada com os protocolos utilizados ${ }^{15}$. Putman et al. ${ }^{16}$ demonstraram que o treinamento concorrente resultou numa maior transição de fibras rápidas para lentas e que também houve uma atenuação da hipertrofia das fibras musculares do tipo I, quando comparado ao treinamento de força isolado. Entretanto, a interação força-endurance parece não ficar prejudicada, havendo, em alguns casos, melhora da performance de indivíduos em atividades aeróbias ${ }^{17,18}$.

Nesse sentido, o objetivo do estudo foi verificar a influência da ordem de exercícios físicos realizados em um único dia nos parâmetros de força, composição corporal e flexibilidade em mulheres adultas, com mais de 50 anos, durante 12 semanas de treinamento.

\section{PROCEDIMENTOS METODOLÓGICOS}

Esse tipo de trabalho caracteriza-se como um estudo experimental com alocação aleatória. O estudo foi realizado nas dependências da Escola Superior de Educação Física (ESEF) da Universidade Federal de Pelotas (UFPel).

Foram selecionados para este estudo 26 indivíduos do gênero feminino, divididos aleatoriamente em dois grupos: 11 sujeitos para o grupo $\mathrm{A}_{1}$ (atividade aeróbia seguida de treinamento com pesos) e 15 sujeitos para o grupo $\mathrm{M}_{1}$ (treinamento com pesos seguido de atividade aeróbia). Foram considerados como critério de exclusão: ser fumantes ou apresentar qualquer tipo de doenças que impedisse a prática regulada das atividades físicas, comprovadas por apresentação de atestado médico. As aulas aconteceram no período da tarde (entre 14:00 e 16:30 h), com intervalo entre as sessões de, no mínimo, 24 horas para a recuperação. As atividades foram ministradas por estagiários do Laboratório de Bioquímica e Fisiologia do Exercício (LABFEX) e supervisionados pelo pesquisador responsável pela pesquisa.

É importante salientar que todos os participantes das intervenções estavam há três meses sem realizar qualquer tipo de atividade física sistematizada. 
Ambos os grupos foram instruídos a manter suas atividades da vida diária (AVDs) e a não realizar qualquer tipo de atividade física sistematizada durante o período do protocolo experimental. Depois de esclarecidos verbalmente sobre os procedimentos aos quais seriam submetidos, os participantes assinaram um termo de consentimento livre e esclarecido. A pesquisa foi aprovada pelo Comitê de Ética em Pesquisa da Faculdade de Educação Física da Universidade Federal de Pelotas, sob protocolo número 021/2008.

\section{Testes}

No início da pesquisa, todos os componentes da amostra foram convidados a se deslocar ao LABFEX da ESEF/UFPel onde foram realizados os pré-testes. A força de membros inferiores e lombar foi medida através de dinamometria em dinamômetro de costas, pernas e peito, da marca Baseline (Estados Unidos), com precisão de $10 \mathrm{Kgf}$ e a força de preensão manual através de dinamômetro de preensão manual, da marca Jamar (Canadá), com precisão de 2 Kgf. A composição corporal foi determinada por medida das dobras cutâneas tricipital, bicipital, subescapular, suprailíaca, abdominal, medial da coxa e medial da perna, com plicômetro científico tipo Harpenden, da marca Cescorf (Brasil), com precisão de $0,1 \mathrm{~cm}$. A flexibilidade foi determinada através do teste de sentar e alcançar de Wells \& Dillon ${ }^{19}$. A carga do treinamento com pesos foi determinada através do teste por repetições de 10 RM.

\section{Protocolo de treinamento}

O programa foi executado durante doze semanas consecutivas, compreendendo três sessões semanais, com duração de uma hora e dez minutos, inclusive nos feriados, ficando estabelecido um limite máximo de três faltas para permanência no programa. $O$ grupo $\mathrm{A}_{1}$ realizou 30 minutos de exercício aeróbio em cicloergômetro e, logo em seguida, uma série de treinamento com pesos constituída de oito exercícios, envolvendo grandes e pequenos grupamentos musculares: supino sentado (peitoral), puxada por trás (grande dorsal), extensão de joelho (quadríceps femoral), flexão de joelho (biceps femoral), rosca bíceps pulley (bíceps braquial), tríceps pulley (tríceps braquial), flexão plantar (panturrilha) e abdominal reto no solo (reto abdominal). $\mathrm{O}$ grupo $\mathrm{M}_{1}$ realizou as mesmas atividades do grupo, citadas anteriormente, somente com a ordem invertida das mesmas (treinamento com pesos seguido de exercício em cicloergômetro). Ambas intervenções eram realizadas no mesmo horário e no mesmo local. Para um melhor entendimento das participantes quanto à ordem de realização das atividades, foram adotadas fichas de cores diferentes para cada grupo.

Após o término das avaliações, foi realizado um período de adaptação com duração de duas semanas para uma melhor assimilação das idosas à ordem e realização dos exercícios. Logo em seguida, deu-se início ao programa, cujas aulas tinham uma hora e dez minutos de duração, com os cinco minutos iniciais e finais destinados, respectivamente, ao aquecimento e volta à calma. $\mathrm{O}$ tempo restante foi dividido, igualmente, entre os treinamentos aeróbio e com pesos. O treinamento com pesos utilizou cargas equivalentes a $50 \%$ do teste de 10 RM, na primeira metade do programa, e $65 \%$, nas últimas seis semanas, quando foram realizadas duas séries para cada exercício, exceto abdominais (duas séries de 50 repetições). Para prescrição da atividade aeróbia, foi utilizada a Escala de Esforço Percebido de Borg (entre 13 e 15$)^{20}$. Os intervalos entre os exercícios que compuseram as séries do treinamento com pesos tinham duração de, aproximadamente, 60 segundos. As atividades foram realizadas em duplas, de maneira que, enquanto uma realizava o exercício a outra descansava.

Ao término das 12 semanas de estudo, os indivíduos foram levados ao LABFEX para a realização dos pós-testes.

\section{Análise estatística}

A análise dos dados foi realizada através da estatística descritiva (média \pm dp) e inferencial (ANOVA oneway). Foi testada a normalidade dos desfechos, de modo a atender os pressupostos da estatística inferencial. Aceitou-se p $<0,05$ como indicador de significância estatística e o pacote estatístico utilizado foi o Stata 9.0.

\section{RESULTADOS}

Foram analisados 26 indivíduos idosos do sexo feminino (11 no grupo $A_{1}$ e 15 no grupo $M_{1}$ ), com idade mínima de 50 anos. Não houve diferença estatística entre as variáveis idade $(\mathrm{p}=0,8)$, peso $(\mathrm{p}=0,1)$, estatura $(\mathrm{p}=0,6)$, índice de massa corporal (IMC) $(\mathrm{p}=0,08)$ e somatório de dobras $(\mathrm{p}=0,07)$ [Tabela 1]. Após doze semanas de treinamento, o grupo $A_{1}$ obteve médias de peso, IMC e somatório de dobras de, respectivamente, $64,5 \mathrm{~kg}(\mathrm{dp}=10,6), 26,4 \mathrm{Kg} /$ $\mathrm{m}^{2}(\mathrm{dp}=4,8)$ e $138,6 \mathrm{~mm}$, sem diferença estatística do pré para o pós-teste. No grupo $\mathrm{M}_{1}$, após as doze semanas de intervenção, os valores médios de peso, 
IMC e somatório de dobras foram de $72,0 \mathrm{Kg}$ (dp $=9,9), 29,7 \mathrm{Kg} / \mathrm{m}^{2}(\mathrm{dp}=3,6)$ e $166 \mathrm{~mm}(\mathrm{dp}=33,6)$, também não apresentando diferença significativa de pré para pós-teste. Quando da realização da comparação das médias pós-intervenção entre os grupos $A_{1}$ e $M_{1}$, verificou-se diferença estatisticamente significativa no IMC $(p=0,05)$ e valores limítrofes $(\mathrm{p}=0,07)$ para as medidas de somatório de dobras e peso corporal, a favor do grupo $\mathrm{M}_{1}$.

Em relação as medidas de força, os grupos $A_{1}$ e $M_{1}$ não apresentaram diferença nas médias iniciais de força da região lombar $(\mathrm{p}=0,9)$, membros inferiores $(p=0,3)$ e preensão manual $(p=0,5)$. $O$ grupo $A_{1}$ apresentou alterações significativas na força da região lombar $(\mathrm{p}=0,01)$ e de membros inferiores $(\mathrm{p}=0,0002)$ após as 12 semanas e o grupo $\mathrm{M}_{1}$ somente na força de membros inferiores $(\mathrm{p}=0,02)$. Quando os resultados dos grupos foram comparados no pós-teste, não se verificou diferença alguma entre as medidas de força estudadas (Tabela 2).

Em relação à flexibilidade, os grupos foram semelhantes no inicio do estudo. Após 12 semanas de treinamento, os níveis de flexibilidade permaneceram inalterados para os grupos $\mathrm{A}_{1}$ e $\mathrm{M}_{1}$. Os níveis de flexibilidade pós-intervenção, entre os grupos, não foram estatisticamente diferentes (Tabela 2).

\section{DISCUSSÃO}

Vários estudos vêm sendo publicados sobre o treinamento de duas ou mais valências físicas, sendo realizadas conjuntamente em uma mesma sessão de trabalho $^{14,21,22}$. Essa temática, apesar de muito descrita, raramente foca grupo de indivíduos com idade eleva- da. Nesse contexto, o presente estudo verificou, dentro do tema treinamento concorrente, se a ordem de execução de trabalho das valências força e exercício aeróbio, pode resultar em diferenças significativas nos índices de força, flexibilidade e composição corporal de mulheres acima dos 50 anos de idade.

Com relação aos resultados das variáveis peso corporal e somatório de dobras cutâneas, o presente estudo concorda com os achados de Viana et al. ${ }^{23}$ os quais mostraram não haver diferenças significativas no componente gordura, em homens adultos, quando comparado o treinamento concorrente com treino de força ou aeróbio, separadamente. $\mathrm{O}$ presente estudo concorda, também, com o relato de Rossato et al..$^{24}$ que, com mulheres adultas, concluiu que a realização de um treinamento combinado de força e endurance, em uma mesma sessão, não é capaz de levar a modificações significativas na composição corporal dessas mulheres.

O IMC, após as 12 semanas de treinamento, apresentou diferença significativa entre os grupos. Estudos atuais demonstram que idosos praticantes de atividades aeróbias conseguem ter uma redução nos valores de peso corporal, levando, consequentemente, a uma redução no IMC. O mesmo não parece acontecer com praticantes de exercícios com pesos isoladamente, pelo aumento da massa muscular, o que pode acarretar na manutenção ou aumento no índice de massa corporal ${ }^{24}$. No presente estudo, os grupos já apresentavam, no inicio do treinamento, diferença limítrofe $(p=0,07)$ no IMC. O alcance da significância estatística para essa diferença veio em consequência de uma redução na média de peso corporal no grupo $A_{1}$, na pós-intervenção. Não fo-

Tabela 1. Caracterização da amostra no início do estudo (médias \pm dp).

\begin{tabular}{cccccc}
\hline & Peso $(\mathrm{kg})$ & Estatura $(\mathrm{cm})$ & Idade $($ anos $)$ & $\mathrm{IMC}\left(\mathrm{Kg} / \mathrm{m}^{2}\right)$ & $\Sigma \mathrm{DC}(\mathrm{mm})$ \\
\hline$M_{1}(\mathrm{n}=15)$ & $71,2 \pm 10,0$ & $155,0 \pm 0,0$ & $64,2 \pm 5,7$ & $29,8 \pm 3,7$ & $179,6 \pm 33,9$ \\
$\mathrm{~A}_{1}(\mathrm{n}=11)$ & $65,2 \pm 10,9$ & $157,0 \pm 0,1$ & $64,9 \pm 8,1$ & $26,7 \pm 4,8$ & $151,3 \pm 42,6$ \\
\hline
\end{tabular}

$\Sigma \mathrm{DC}=$ somatório de dobras cutâneas

Tabela 2. Médias e desvio padrão das variáveis dependentes força de membros inferiores, força lombar, força de preensão manual, flexibilidade, somatório de dobras cutâneas.

\begin{tabular}{ccccc}
\hline & & $M_{1}$ & & $A_{1}$ \\
\cline { 2 - 5 } & Pré & Pós & Pré & $50,0 \pm 13,0^{*}$ \\
\hline $\begin{array}{c}\text { Força de membros } \\
\text { inferiores (Kgf) }\end{array}$ & $32,8 \pm 12,2$ & $42,9 \pm 10,5^{\#}$ & $28,2 \pm 8,9$ & $53,4 \pm 9,1^{* *}$ \\
$\begin{array}{c}\text { Força lombar (Kgf) } \\
\text { Força de preensão } \\
\text { manual (Kgf) }\end{array}$ & $42,7 \pm 9,7$ & $48,3 \pm 8,2$ & $42,3 \pm 8,2$ & $28,9 \pm 10,8$ \\
$\begin{array}{c}\text { Flexibilidade (cm) } \\
\Sigma \text { DC (mm) }\end{array}$ & $23,9 \pm 6,2$ & $26,1 \pm 5,9$ & $25,8 \pm 6,8$ & $17,8 \pm 11,1$ \\
\hline
\end{tabular}

\# $\mathrm{p}=0,02$ (entre pré- e pós-teste de $\mathrm{M}_{1}$ ) $* \mathrm{p}=0,0002$ (entre pré- e pós-teste de $\mathrm{A}_{1}$ ) ${ }^{* *} \mathrm{p}=0,02$ (entre pré- e pós-teste de $\mathrm{A}_{1}$ ) 
ram controlados aspectos relacionados à dieta dos sujeitos, o que pode ser uma das explicações para a redução do peso no grupo $\mathrm{A}_{1}$.

Alteração nos níveis de força da região lombar do pré-para o pós-teste foi observada no grupo $A_{1}$ e nos níveis de força de membros inferiores em ambos os grupos. Apesar disso, não houve diferença nos ganhos dos níveis de força entre os grupos após as 12 semanas. Brandon et al..$^{25}$, observaram aumento da força máxima de membros inferiores, após 16 semanas de treinamento com pesos, de 40,4\% e $44,7 \%$, respectivamente, para extensão e flexão de joelhos. A melhora da força de membros inferiores, em ambos os grupos, pode estar relacionado, além do trabalho realizado na musculação, com o trabalho aeróbio em cicloergômetro. Estudo de Macaluso et $a .^{26}$ com idosas acima de 65 anos, mostrou que um programa de treinamento realizado em cicloergômetro, independente da velocidade de pedalada, trouxe melhoras no aumento da potência e força de membros inferiores destas idosas.

$O$ presente estudo encontrou melhora da força lombar no grupo que praticou atividade aeróbia e posteriormente musculação. Esse resultado contraria os achados de Souza et al. ${ }^{[21]}$ os quais mostraram que a realização de exercícios aeróbios anteriores aos de força não são capazes de alterar os índices de força máxima dessa região corporal. Contudo, a maioria dos estudos que procuram verificar a influência do componente aeróbio no de força demonstram que há prejuízo no grau de força de membros inferiores ${ }^{27}$.

Os níveis de flexibilidade para os grupos $\mathrm{A}_{1} \mathrm{e}$ $M_{1}$ antes e depois e entre eles, ao final do estudo, permaneceram inalterados. Rebelato et al. ${ }^{28}$ não encontraram diferença nos níveis de flexibilidade de mulheres idosas que participaram de um programa de condicionamento físico, envolvendo trabalhos de força, resistência aeróbia, coordenação e alongamento em uma mesma sessão. A não alteração nos níveis de flexibilidade em nosso estudo pode estar relacionada ao pouco tempo de trabalho despendido a essa valência no treino. Além disso, o instrumento utilizado para a medição da mesma (banco de Wells) parece dificultar a medida em indivíduos idosos. Segundo Shepard ${ }^{29}$, durante a vida ativa, adultos perdem em torno de 8 a 10 centímetros de flexibilidade na região lombar e no quadril, quando medido por meio do teste de alcance máximo "sit and reach". Dentre os vários fatores que colaboram para isso estão a maior rigidez de tendões, ligamentos e cápsulas articulares, devido às deficiências de colágeno. Por outro lado, a manutenção dos valores iniciais é um aspecto que não pode ser desprezado quando se focaliza a qualidade de vida e a independência funcional dos indivíduos mais velhos e a vinculação da força muscular e da flexibilidade corporal com a probabilidade de quedas e lesões músculo-esqueléticas ${ }^{28}$. Em relação a esse fator, Okuma ${ }^{30}$ aponta que, para realizar suas tarefas cotidianas como subir escadas, carregar suas compras e abaixar-se, um idoso necessita de pouca aptidão cardiovascular e muito mais de um conjunto de capacidades como força muscular, resistência muscular localizada e flexibilidade, conjunto este denominado de "aptidão muscular".

O tamanho amostral (que está relacionado ao poder do estudo) e o não controle nutricional das participantes do programa de treinamento (possível variável de confusão) foram as principais limitações encontradas no estudo.

\section{CONCLUSÃO}

No presente estudo, a utilização da ordem de execução dos exercícios aeróbios e com pesos, em uma mesma sessão de treinamento, mostrou alterar, significativamente, a força lombar em $\mathrm{A}_{1}$ e de membros inferiores em $A_{1}$ e $M_{1}$. Além disso, a realização de atividades aeróbias anteriormente as de força levaram a uma maior redução do peso corporal, o que influenciou na diferença encontrada no índice de massa corporal ao final do estudo. Independentemente da ordem de execução dos exercícios, no treinamento concorrente, as mulheres estudadas mantiveram inalterados sua força de preensão manual e os índices de flexibilidade, o que, para pessoas em idade avançada, é de fundamental importância, pois podem afetar diretamente a melhoria da qualidade de vida e a saúde dessa população. Sugere-se que sejam realizados novos estudos com a utilização de um maior tempo de intervenção, no sentido de se tentar obter informações mais precisas acerca desse tipo de treinamento, ainda pouco pesquisado em mulheres nessa faixa etária.

\section{REFERÊNCIAS BIBLIOGRÁFICAS}

1. Gutteridge JM, Halliwell B. Free radicals and antioxidants in the year 2000: a historical look to the future. Ann NY Acad Sci 2000;899:136-147.

2. Baumgartnen R, Koehler K, Gallagher D, Romero L, Heymstleld S, Ross R, et al. Epidemiology of sarcopenia among the elderly in New Mexico. Am J Epidemiol 1988;147(8):755-763.

3. Nahas MV. Atividade física, saúde e qualidade de vida. Londrina: Midiograf; 2003. 
4. Carter ND, Kannus P, Khan KM. Exercise in the prevention of falls in older people: a systematic literature review examining the rationale and the evidence. Sports Med 2001;31(6):427-438.

5. Liu-Ambrose T, Khan KM, Eng JJ, Janssen PA, Lord SR, McKay HA. Resistance and agility training reduce fall risk in women aged 75 to 85 with low bone mass: a 6-month randomized, controlled trial. J Am Geriatr Soc 2004;52(5):1-9.

6. Matsudo SM, Matsudo VKR, Neto TLB. Impacto do envelhecimento nas variáveis antropométricas, neuromotoras e metabólicas da aptidão física. Rev Bras Ciên Mov 2000;8(4):21-32.

7. Fleck S, Kraemer WJ. Fundamentos do treinamento de força muscular. Porto Alegre: Artmed; 1999.

8. LaCroix AZ, Leveille SG, Hecht JA, Grothaus LC, Wagner EH. Does walking decrease the risk of cardiovascular disease hospitalizations and death in older adults? J Am Geriatr Soc 1996;44(2):113-120.

9. Keysor JJ, Jette AM. Have we oversold the benefit of late-life exercise? J Gerontol A Bio Sci Med Sci 2001;56(7):M412-423.

10. Haskell W, Lee I, Pate R, Powell K, Blair S, Franklin B, et al. American College of Sports Medicine; American Heart Association. Physical activity and public health: updated recommendation for adults from the American College of Sports Medicine and the American Heart Association. Circulation 2007;116(9):1081-1093.

11. Bell GJ, Syrotuik D, Martin TP, Burnham R, Quinney $\mathrm{H}$. Effect of strength training and endurance training on skeletal muscle properties and hormone concentrations in humans. Eur J Appl Physiol 2000;81(5):418-427.

12. Hennessy LC, Watson AWS. The interference effect of training for strength and endurance simultaneously. J Strenght Cond Res 1994;8(1):12-19.

13. Kraemer WJ, Patton JF, Gordon SE. Compatibility of high intensity strength and endurance training on hormonal and skeletal muscle adaptations. J Appl Physiol 1995;78(3):976-989.

14. Leveritt M, Abernethy PJ, Barry BK, Logan PA. Concurrent strength and endurance training: a review. Sports Med 1999;28(6):413-427.

15. Guedes DP. Musculação: estética e saúde feminina. Rio de Janeiro: Phorte; 2003.

16. Putman CT, Xu X, Gillies E, Maclean IM, BellL GJ. Effects of strength, endurance and combined training on myosin heavy chain content and fiber type distribution in humans. Eur J Appl Physiol 2004;92(4):376-384.

17. Paavolainen L, Hakkinen K, Hamalainen I, Nummela A, Rusko H. Explosive-strength training improves $5-\mathrm{km}$ running time by improving running economy and muscle power. J Appl Physiol 1999;86(5):1527-1533.

18. Millet P, Jaquen B, Borrani F, Candau R. Effects of concurrent endurance and strength training on running economy and VO2 kinetics. Med Sci Sports Exerc 2002;34(8):1351-1359.
19. Marley WP, Linnerud AC. A three-year study of the Astrand-Ryhming step test. Res Quart Exerc Sport 1976;47(2):211-217.

20. Borg G. Psychophysical basis of perceived exertion. Med Sci Sports Exerc 1982;14(5):377-381.

21. de Souza EO, Tricoli V, Franchini E, Paulo AC, Regazzini M, Ugrinowitsch C. Acute effect of two aerobic exercise modes on maximum strength endurance. J Strenght Cond Res 2007;21(4):1286-1290.

22. Leveritt M, Abernethy PJ, Barry BK, Logan PA. Concurrent strength and endurance training the influence of dependent variable selection. J Strenght Cond Res 2003;17(3):503-508.

23. Viana MV, Filho JF, Dantas EHM, Perez AJ. Efeitos de um programa de exercícios físicos concorrentes sobre a massa muscular, a potência aeróbica e a composição corporal em adultos aeróbicos e anaeróbicos. Fit Perf J 2007;6(3):135-139.

24. Rossato M, Binotto MA, Roth MA, Temp H, Carpes FP, Alonso JL, et al. Efeito de um treinamento combinado de força e endurance sobre componentes corporais de mulheres na fase de perimenopausa. Rev Port Cien Desp 2007;7(1):92-99.

25. Brandon LJ, Boyette LW, Gaasch DA, Lloyd A. Effects of lower extremity strength training on functional mobility in older adults. J Aging Phys Act 2000;8(3):214-227.

26. Macaluso A, Young A, Gibb KS, Rowe DA, De Vito G. Cycling as a novel approach to resistance training increases muscle strength, power, and selected functional abilities in healthy older women. J Appl Physiol 2003;95(6):2544-2553.

27. Sporer B, Wenger H. Effects of aerobic exercise on strength performance following various periods of recovery. J Strenght Cond Res 2003;17(4):188-192.

28. Rebelatto JR, Calvo JI, Orejuela JR, Portillo JC. Influência de um programa de atividade física de longa duração sobre a força muscular manual e a flexibilidade corporal de mulheres idosas. Rev Bras Fisioter 2006;10(1):127-132.

29. Shephard RJ. Aging and exercise. Sportscience 1998; Available from: <http:// sportsci.org.> [2008 May 30].

30. Okuma SS. O idoso e a atividade física: fundamentos e pesquisa. Campinas: Papirus; 2002.
Endereço para correspondência
Marcelo Cozzensa da Silva
Rua Luiz de Camões, 625. Bairro Areal
CEP: 95055-630. Pelotas, RS. Brasil.
E-mail: cozzensa@terra.com.br 